\title{
Quasi pseudo-inverse reconstruction for rotating PET scanners
}

\author{
Javier Sánchez-González, Samuel España, Mónica Abella, Juan José Vaquero, Senior Member, IEEE, \\ Eduardo Lage, Javier Pascau, Member, IEEE, Manuel Desco.
}

\begin{abstract}
A new reconstruction strategy is proposed for $3 \mathrm{D}$ images acquired with rotating PET scanners. This method is based on the decomposition of the System Response Matrix (SRM) into a projection and a rotation components that are inverted independently. We present the rationale of the new reconstruction procedure as well as some examples to compare with analytical 3D reconstruction (3DRP) and 3D statistical reconstruction (3D-OSEM).
\end{abstract}

Index Terms Statistical image reconstruction, Positron Emisssion Tomography, Small animal imaging.

\section{INTRODUCTION}

Some architectures of high resolution small animal PET $N_{\text {scanners are based on a determined number of opposite }}$ rotating detectors, that acquire the annihilations events emitted by the sample.

The reconstruction problem in PET is to determine the map of annihilations given the data gathered by pairs of the detectors. The image reconstruction process for PET imaging can be formulated in matrix notation as:

$$
E \rho=m,
$$

where $\mathbf{E}$ is a matrix $\left(n_{d} \mathrm{x} n_{p} ; n_{d}\right.$ number of lines of response and $n_{p}$ number of pixels) that represents the system response matrix (SRM), where each element of $\mathbf{E}(i, j)$ is defined as the probability of detecting an annihilation event emitted from pixel $j$ by a line of response $i$. $\rho$ is a $\left(1 \times n_{p}\right)$ column vector that contains all the pixels of the image and $\mathbf{m}$ is another $(1 \mathrm{x}$ $n_{d}$ ) column vector that represents the whole acquired data set.

For a conventional PET acquisition, where there are thousand of lines of response, the size of the SRM makes impossible a direct solution of (1), requiring iterative algorithms as EM [1] or OSEM [2] to obtain the reconstructed image. One of the main drawbacks of these iterative methods

Manuscript received September 5, 2005. Part of this work is founded by the IM3 network (G03/185 Ministerio de Sanidad), with grants from the Ministerio de Educación y Ciencia, project TEC2004 07052 C02 01, and Ministerio de Industria, Turismo y Comercio project FIT 33010120043.

J. Sánchez is with the Unidad de Medicina y Cirugía Experimental, Hospital GU Gregorio Marañón, Madrid, Spain (corresponding author phone: +34 91426 5067; fax: +34 91426 5067; e mail: jsanchez@mce.hggm.es).

S. España is with Dpto. Física Atómica, Molecular y Nuclear, Universidad Complutense de Madrid.

M. Abella, JJ. Vaquero, E. Lage, J. Pascau and M. Desco are with the Unidad de Medicina y Cirugía Experimental, Hospital GU Gregorio Marañón, Madrid, Spain is their computational burden. To solve this problem some authors have proposed to pre-calculate the singular value decomposition (SVD) of the whole SRM [3, 4], obtaining a reconstructed image in a computation time comparable to that of the FBP algorithm [4]. The problem of these methods is the size of the matrix to invert, which makes it difficult to apply to $3 \mathrm{D}$ reconstruction.

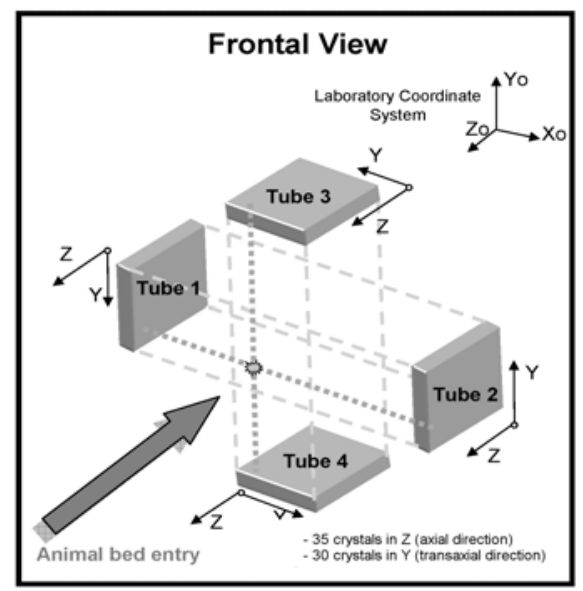

Figure 1: Diagram of a rotating PET scanner .

\section{THEORY}

The SRM for scanner based on opposite gamma radiation detectors (Fig. 1) can be decomposed into two different components: the projection of the lines of response at a fixed gantry position and the gantry rotation. From a mathematical point of view, the SRM can be expressed as:

$$
\mathbf{E}=\left[\begin{array}{ll}
\mathbf{I}_{\varphi} \otimes \mathbf{P}
\end{array}\right]\left[\left(\begin{array}{c}
\mathbf{R}\left(\alpha_{1}\right) \\
\mathbf{R}\left(\alpha_{2}\right) \\
\vdots \\
\mathbf{R}\left(\alpha_{i}\right)
\end{array}\right)\right] \text {, }
$$

where the $\mathbf{P}$ is a matrix $\left(n_{c} \times n_{p} ; n_{c}\right.$ is the number of lines of response between opposite detectors) that represents the projection process for all possible lines of response for a given gantry position; $\mathbf{R}\left(\alpha_{i}\right)$ is a $\left(n_{p} \times n_{p}\right)$ matrix that represents the rotation of the gantry an $\alpha_{i}$ angle, $\mathrm{I}_{\varphi}$ is a $\left(n_{\alpha} \mathrm{x} n_{\alpha ;} n_{\alpha}\right.$ number of rotating angles) identity matrix and $\otimes$ represents the direct product of matrices.

The SVD solution of a conventional 3D PET experiment 
taking into account all possible lines of response and $n_{\alpha}$ angles implies to obtain the SVD of a matrix of a size of $n_{\alpha} n_{c} \mathrm{x} n_{\alpha} n_{c}$ elements. For a conventional study, the matrix to be inverted has thus a huge dimension. However, a faster inversion of equation (2) could be achieved as:

$$
\mathbf{E}^{1}=\frac{1}{n_{\varphi}}\left[\left(\begin{array}{c}
\mathbf{R}\left(-\alpha_{1}\right) \\
\mathbf{R}\left(-\alpha_{2}\right) \\
\vdots \\
\mathbf{R}\left(-\alpha_{i}\right)
\end{array}\right)\right]^{\top}\left[\mathbf{l}_{\varphi} \otimes \mathbf{P}^{\dagger}\right]
$$

where $\mathbf{P}^{\dagger}$ represent the pseudo-inverse of the $\mathbf{P}$ matrix [5] for under-determined systems, obtained as:

$$
\mathbf{P}^{\dagger}=\mathbf{P}^{\top}\left(\mathbf{P} \mathbf{P}^{\top}\right)^{\dagger}
$$

The inverse of the $\mathbf{P P}^{\top}$ can be estimated by singular value decomposition

$$
\left(\mathbf{P}^{\top} \mathbf{P}\right)^{\dagger}=\mathbf{U} \boldsymbol{\Sigma}^{\dagger} \mathbf{U}^{\top}
$$

where $\boldsymbol{\Sigma}^{\dagger}$ is a diagonal matrix with diagonal element given by

$$
\boldsymbol{\Sigma}_{i}^{\top}=\sigma_{i} /\left(\sigma_{i}^{2}+\sigma_{0}^{2} \beta\right),
$$

where $\sigma_{\mathrm{i}}$ are the singular values of the $\mathbf{P P}^{\top}$ and $\beta$ is a regularization parameter to control noise amplification in the reconstructed image.

The second expression in the right side of equation (3) implies to calculate the inversion of the rotation component. This inverse matrix can be obtained concatenating all the rotation matrices in a row format with a rotation angle equal to minus $\alpha_{i}$.

Equation (3) represents a possible inverse of equation (2) although it does not ensure the minimum-norm solution of (1) as the problem is analyzed independently. However, this solution implies the inversion of each component (projection and rotation) independently, reducing noticeably the size of the matrices to invert and the computation requirements.

The new reconstruction strategy can be summarized in three steps (Fig. 2):

1. Data for each angle are multiplied by the pseudoinverse of the projection matrix, obtaining an estimated image of the object.

2. $\quad$ Each estimated image is rotated an angle $-\alpha_{i}$, where $i$ represents the different gantry positions during the acquisition (second column of Fig. 2).

3. All the estimated images obtained in the step 2 are added to obtain the final reconstructed image (third column of Fig. 2).

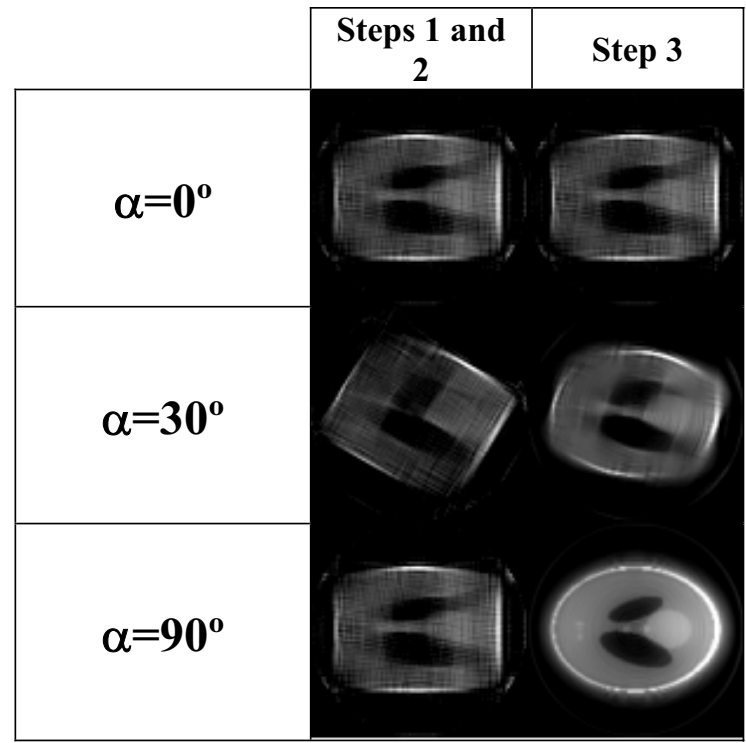

Figure 2: Results of the proposed procedure for step 1, 2 and 3 at three different instance of the image reconstruction.

This description of the reconstruction procedure resembles a filtered backprojection reconstruction, where the filter is obtained from the problem analytical. In our case the pseudoinverse of the projection matrix can be seen as a filter that can include the prior knowledge of the system.

\section{MATERIAL AND METHODS}

\section{A. $\mathbf{P}$ matrix estimation}

The matrix $\mathbf{P}$ was estimated by Monte Carlo simulation from the LORs corresponding to three crystals in the $\mathrm{Z}$ direction and 30 crystals in the $\mathrm{Y}$ direction (Fig 1), thus yielding $(3 * 30)=8100$ LORs per detectors pair. These LOR comprise a volume of $115 \times 115 \times 6$ pixels in $\mathrm{X}, \mathrm{Y}$ and $\mathrm{Z}$ direction, respectively. To reconstruct the whole volume, the matrix $\mathbf{P}^{\dagger}$ was multiplied by the data that correspond to each group of three crystals along the $\mathrm{Z}$ direction, covering a whole volume of $115 \times 115 \times 70$ pixels.

To get a good estimation of pixels probabilities across the field of view, the Monte Carlo simulation took into account the properties of the scintillator crystals as well as Compton and positron range effects.

\section{B. Acquired data}

To evaluate the new reconstruction procedure we used data from a hot Derenzo-like phantom, acquired in an rPET scanner (Suinsa Medical Systems Inc, Madrid, Spain) (Fig. 3).

During the experiment, more than $1.5 \mathrm{E} 8$ events were collected to achieve a good signal to noise ratio. The data were acquired in list mode during the continuous rotation of the gantry. The events were organized as histograms of LOR with and angular binning of one degree. The data were corrected from decay, energy, crystals sensitivities and angular exposition.

To assess the new reconstruction method, the same data set was also reconstructed with 3D-OSEM (3 iterations and 25 
subsets) and 3DRP (Colsher filter and $\mathrm{Z}$ maximum axial difference of 17 crystals). These two reconstruction methods use the data organized as a sinogram to reduce the number of equations in the reconstruction process. The sinogram was created from the list mode with a dimension of ( $\left.\rho, \theta, Z_{1}, Z_{2}\right)$ $(59,180,35,35)$.

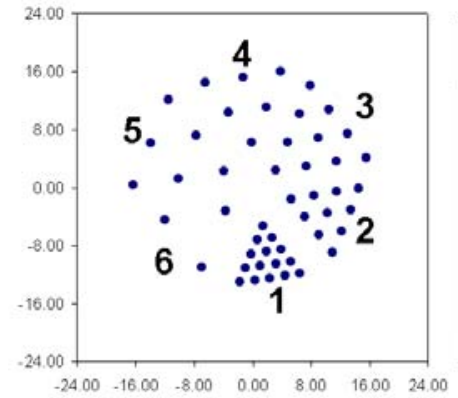

\begin{tabular}{|c|c|c|}
\hline Sector & $\begin{array}{c}\text { Separation } \\
(\mathbf{m m})\end{array}$ & $\begin{array}{c}\text { Dimeter } \\
(\mathbf{m m})\end{array}$ \\
\hline 1 & 2 & 0.50 \\
2 & 3 & 0.75 \\
3 & 4 & 1.00 \\
4 & 5 & 1.25 \\
5 & 6 & 1.50 \\
6 & 8 & 2.00 \\
\hline
\end{tabular}

Figure 3: Diagram of hot Derenzo-like phantom acquired with rPET scanner and the dimensions of each segment.
The computation time for the 3DRP (with a maximum axial difference of 17) was $5 \mathrm{~min}$, for 3D-OSEM (3 iterations, 25 subsets) was $60 \mathrm{~min}$ and for the new reconstruction procedure was $25 \mathrm{~min}$. Our procedure needs about half the reconstruction time than 3D-OSEM, independently of the regularization parameter $\beta$.

\section{DISCUSSION AND CONCLUSIONS}

This work proposes a new reconstruction procedure for 3DPET studies from rotating scanners. The proposed reconstruction exploits the modularity of the SRM to reduce the reconstruction complexity.

Our results with a hot Derenzo-like phantom showed that the proposed procedure produces similar results to those obtained with 3D-OSEM in terms of resolution, while reducing the computation burden by a factor of two.

\begin{tabular}{|c|c|c|c|c|}
\hline & $\begin{array}{c}\text { 3DRP } \\
\text { Colsher filter, } 17 \text { axial dif, }\end{array}$ & $\begin{array}{c}\text { 3D-OSEM } \\
3 \text { Iterations, } 25 \text { subsets }\end{array}$ & $\begin{array}{c}\text { Quasi pseudo-inverse } \\
\beta 1 \mathrm{E} 4\end{array}$ & $\begin{array}{c}\text { Quasi pseudo-inverse } \\
\beta 1 \mathrm{E} 5\end{array}$ \\
\hline $\begin{array}{l}\text { Sum of all } \\
\text { the slices } \\
\text { in } Z \\
\text { direction }\end{array}$ & 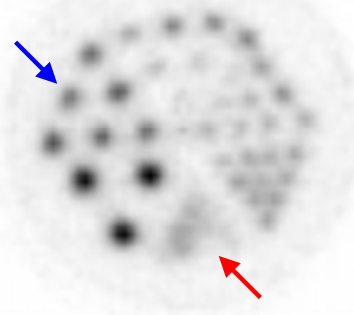 & 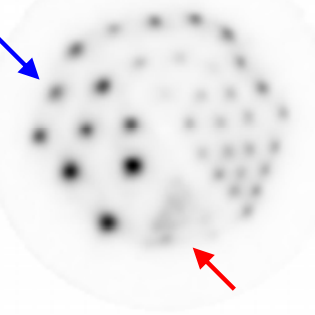 & 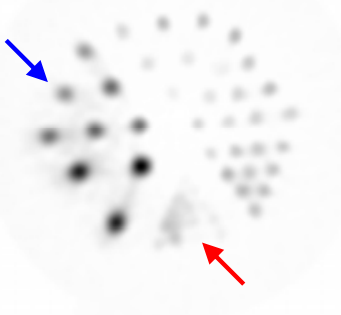 & 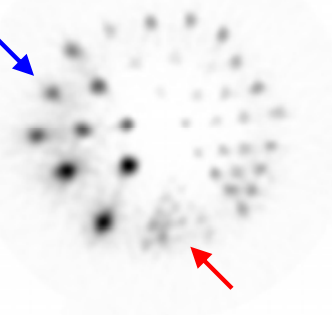 \\
\hline
\end{tabular}

Figure 4: Reconstruction results of the proposed method and 3DRP and 3D-OSEM reconstruction methods.

\section{RESUlts}

Images in Fig 4 show the result yielded by the three reconstruction methods. All of them show similar images, although 3D-OSEM and our reconstruction method produced better results than 3DRP, probably due to the prior information included in the reconstruction process.

The result of 3D-OSEM and our reconstruction method with $\beta=1 \mathrm{E}-4$ provide equivalent results. However, our reconstruction with $\beta=1 \mathrm{E}-5$ can resolve the smallest points of the phantom (red arrows, Fig. 4). This difference can derive from the fact that the 3D-OSEM method uses data organized as sinograms to reduce the size of the SRM. The inclusion of data from different crystals in the same LOR of the sinogram spreads the probabilities along the FOV, masking the smallest points of the phantom.

Smaller values of the parameter $\beta$ increase the noise in the final reconstructed image (blue arrows in Fig. 4). This effect is explained by the fact that smaller values of $\beta$, following equation (6), imply to use smaller singular values which play a more important role in the reconstruction process. The inclusion of these singular values generates a better representation of the data, albeit producing noise amplification
A drawback of the proposed procedure is that SVD inversion of the $\mathbf{P}^{\mathrm{T}} \mathbf{P}$ matrix assumes that data are corrupted by Gaussian noise, which does not necessarily holds in our case. This problem deserves further analysis to determine the effect of using a different distribution in the statistical approach.

\section{ACKNOWLEDGEMENT}

The authors thank Dr. J Pavia CIME (CETIR Group Mèdic, Barcelona) for providing the phantom data acquired in the rPET system and SUINSA Medical Systems for their support.

\section{REFERENCES}

[1] L. A. Shepp and Y. Vardi, "Maximum likelihood estimation for emission tomography.," IEEE Trans Med Imaging, vol. MI-1, pp. 113-121, 1982.

[2] H. M. Hudson and R. S. Larkin, "Accelerated image reconstruction using ordered subsets of projection data.," IEEE Trans Med Imaging, vol. 13, pp. 601609, 1994.

[3] V. V. Selivanov and R. Lecomte, "Fast PET image reconstruction based on SVD decomposition of the system matrix.," IEEE Trans. Nucl. Sci., vol. 48, pp. 761-767, 2001. 
[4] T. Yamaya, T. Obi, M. Yamaguchi, K. Kita, N. Ohyama, and H. Murayama, "Algebraic PET image reconstruction with pre-computed reconstruction operators using subsets of sensitivity functions.," IEEE Trans. Nucl. Sci., vol. 47, pp. 1670-1675, 2000.

[5] G. H. Golub and C. F. Van Loan, Matrix computations, 3 ed. Baltimore: Johns Hopkins University Press, 1996. 